\title{
Treatment of Typhoid Fever: a Comparative Trial of Ampicillin and Chloramphenicol
}

\author{
W. L. SANDERS,* M.D., M.R.C.P
}

Brit. med. F., 1965, 2, 1226-1227

This paper is a study of the relative efficacy of ampicillin (Penbritin) and chloramphenicol in the treatment of typhoid fever. A series of 40 patients (20 in each group) were treated in the Sungei Gerong hospital of P.T. Stanvac. The patients, all Indonesian, were allotted at random to either group. Their ages ranged from $2 \frac{1}{2}$ to 38 years. The age distribution is shown in Table I.

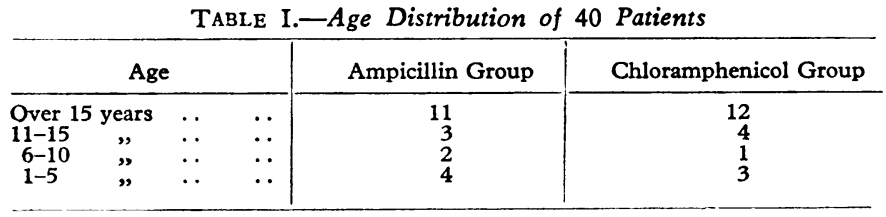

The average time interval from the onset of the illness to the start of treatment in the ampicillin group was 7.7 days, range 2 to 14 days, and in the chloramphenicol group 7.3 days, range 2 to 21 days.

On clinical grounds 10 of the ampicillin group and 7 of the chloramphenicol group were regarded as severe cases.

\section{Dosage and Follow-up}

Dosage.-In patients aged over 10 years ampicillin was given in a dose of $1 \mathrm{~g}$. six-hourly; in those aged 6-10 $500 \mathrm{mg}$. sixhourly ; and in those aged 1-5 $250 \mathrm{mg}$. six-hourly. In the corresponding age groups chloramphenicol was given in a dose of $500 \mathrm{mg}$. six-hourly, reducing to $250 \mathrm{mg}$. when the temperature had fallen to normal ; $250 \mathrm{mg}$. six-hourly ; and $125 \mathrm{mg}$. six-hourly. These doses are high when it is considered that the average weight of an adult male Indonesian is $50 \mathrm{~kg}$. Treatment in all cases was continued for 14 days.

On admission all patients had routine white-cell counts performed and blood films for malaria taken. One patient in the ampicillin group was found to have vivax malaria.

Samples for blood culture were taken on three successive days. Samples for the Widal reaction were taken on admission, and at weekly intervals. Stool cultures were made on admission, at weekly intervals, and on three successive days at the end of treatment.

All patients were followed up at intervals varying from two to six weeks after discharge from hospital, when three further stool cultures were made. It was possible to test the in-vitro sensitivity of the organism in only two cases; both were fully sensitive to ampicillin; disks of $25 \mu \mathrm{g}$. were used.

\section{Diagnosis}

Positive blood cultures were accepted as proof of the diagnosis. Positive stool cultures were also considered as diagnostic when they supported the clinical findings. Some of the patients had previously been inoculated with T.A.B. and some had not, and it was not always possible to determine whether a

*. Specialist Physician and Medical Director, P.T. Stanvac Indonesia, Sungei Gerong, Indonesia. given patient had been so inoculated. Under these circumstances, taken in conjunction with the clinical findings, a Widal reaction positive in the $O$ agglutinin to a titre of 1 in 160 was regarded as diagnostic. The numbers of patients diagnosed by each method is shown in Table II.

In all cases treatment was started as soon as the clinical diagnosis was made and the first blood sample had been taken: this may account for the relatively low incidence of positive blood cultures.

\begin{tabular}{|c|c|c|}
\hline & Ampicillin Group & Chloramphenicol Group \\
\hline $\begin{array}{l}\text { Positive blood culture } \ldots \\
\text { Positive stool culture } \\
\text { Positive Widal } \ldots\end{array}$ & $\begin{array}{r}10 \\
5 \\
5\end{array}$ & $\begin{array}{l}8 \\
3 \\
9\end{array}$ \\
\hline
\end{tabular}

\section{Findings}

Of the 10 cases in the ampicillin group which were regarded as severe six were changed to treatment with chloramphenicol because their condition was worsening after periods of treatment with ampicillin varying from four to eight days. After the change to chloramphenicol the temperature in these cases fell to normal in an average of four days-range three to six days. In the remaining four severe cases, in which treatment with ampicillin was continued, the temperature fell to normal in an average of 7.5 days-range 5 to 11 days.

In the 10 cases of moderate severity in the ampicillin group the temperatures fell to normal in an average of three daysrange one to seven days.

In the seven severe cases in the chloramphenicol group the temperatures fell to normal in an average of 3.7 days-range two to six days. In the 13 cases of moderate severity in this group the temperature fell to normal in an average of 2.7 daysrange one to five days.

There was one relapse in the ampicillin group. This case failed to respond to a further five days' treatment with ampicillin, but the temperature fell to normal after three days' treatment with chloramphenicol.

Two relapses occurred in the chloramphenicol group. One of these cases was treated with a further course of chloramphenicol, and the temperature fell after five days' treatment ; the other was treated with a course of ampicillin, when the temperature fell to normal after two days.

Stool cultures were positive at the end of treatment in two cases in the ampicillin group but in none of the chloramphenicol group. These two cases were treated with a further course of ampicillin and follow-up stools were negative. At follow-up after discharge from hospital stool cultures were positive in one patient from each group. Both were treated with courses of ampicillin, and further follow-up stools were negative.

Two patients developed side-effects while under treatment with ampicillin. One had a generalized macular rash on the tenth day of treatment which persisted until ampicillin was withdrawn on the twelfth day ; the other developed a fever of $40^{\circ} \mathrm{C}$. on the eighth day of treatment which persisted until the 
drug was withdrawn on the twelfth day. There were no sideeffects in the chloramphenicol group.

\section{Discussion}

These results show that in the treatment of severe cases of typhoid fever chloramphenicol is more effective than ampicillin as judged by the length of time taken for the temperature to subside. Further, in this series the response to chloramphenicol was uniformly good irrespective of the severity of the illness. This conclusion supports that of Patel (1964). In contrast, in six cases the treatment had to be changed to chloramphenicol because of apparent lack of response to ampicillin. It could be argued that in these cases treatment with ampicillin was not continued long enough, but in view of the worsening clinical condition of these patients it was not thought justifiable to continue.

The time taken for the temperature to subside in severe cases treated with ampicillin in this series agrees with the figures of Uwaydah and Shamma'a (1964); but those authors do not describe the severity of their cases.
Patel (1964) states that in patients treated with ampicillin the duration of illness before the start of treatment had a great effect on the response to the drug. This was not noted in the present series, possibly because on average the time interval before treatment was shorter than in Patel's series.

The results in cases of moderate severity show little difference between the two drugs.

\section{Summary}

A comparative trial of ampicillin and chloramphenicol in the treatment of typhoid fever is described in a total of 40 patients.

The conclusion is reached that chloramphenicol is superior to ampicillin in the treatment of severe typhoid fever.

I wish to thank Dr. O. P. W. Robinson, of Beecham Research Laboratories, for generous supplies of Penbritin.

\section{REFERENCES}

Patel, K. M. (1964). Third International Congress of Chemotherapy, Stuttgart, 1963, vol. 1, p. 416. Thieme, Stuttgart Uwaydah, M., and Shamma'a. M. (1964). Lancet, 1, 1242.

\section{Medical Memoranda}

\section{Systemic Lupus Erythematosus and Pregnancy}

\section{Brit. med. F., 1965, 2, 1227-1228}

This memorandum reports a case of systemic lupus erythematosus of pregnancy and reviews the literature with special reference to treatment.

\section{CASE Report}

A patient, now aged 42 years, was first admitted to the medical unit in April 1957 with systemic lupus erythematosus. She gave a history of increasing breathlessness for four years which had become worse over the last month before admission. At this time she was orthopnoeic and cyanosed, with clinical evidence of atelectasis of the left base of the lung. There was inactive arthritic involvement of the hands and wrists. The diagnosis of systemic lupus erythematosus was confirmed by finding numerous lupus erythematosus cells in the blood. She was treated with steroids with considerable improvement in her effort dyspnoea. Since then she has been admitted on numerous occasions with exacerbation of her joint and muscle pains, increasing effort dyspnoea, and one episode of left-sided pleuritic chest pain. During this time har prednisolone dosage was altered according to the activity of the disease-the dosage varying between 15 to $75 \mathrm{mg}$. daily.

She attended the medical out-patients department on 28 March 1963 with a history of amenorrhoea since 1 August 1962 and complaining that her abdomen was getting bigger. The uterus was found to be enlarged to approximately 22 weeks' pregnancy. She was referred to the obstetric department. The dyspnoea became worse during the first trimester of pregnancy and improved during the middle trimester; it worsened again during the 35th to 36th week of gestation, when she was admitted to hospital. At this time there was evidence of pre-eclamptic toxaemia, the blood-pressure having risen from $120 / 80 \mathrm{~mm}$. $\mathrm{Hg}$ to $130 / 90$ $\mathrm{mm} . \mathrm{Hg}$, and there was a trace of albumin in the urine. These symptoms improved with rest. Labour commenced spontaneously at the 38th week of pregnancy: the second stage was shortened by an outlet forceps delivery while the patient was in the upright position, since dyspnoea became intolerable on lying flat.

A live male infant (birth weight $5 \mathrm{lb} .14 \mathrm{oz} .(2.67 \mathrm{~kg}$.)) was delivered. Nothing abnormal was found on clinical examination. Three weeks after delivery her effort dyspnoea became worse, but there was no evidence of congestive cardiac failure and the bloodpressure had returned to $120 / 80 \mathrm{~mm}$. $\mathrm{Hg}$. Prednisolone was increased to $25 \mathrm{mg}$. daily for the first two weeks post partum; the effort dyspnoea then became less and the prednisolone dosage was reduced to $15 \mathrm{mg}$. daily.

The patient has been observed in the out-patient department for 10 months after delivery and there has been no evidence of deterioration in her condition. The disease is being kept under control with prednisolone $15 \mathrm{mg}$. daily. The baby is alive and well and continues to make satisfactory progress.

The results of recent investigations are as follows: L.E. cells, occasionally present ; haemoglobin, $70 \%$; white blood count, 6,000 differential normal ; E.S.R., 10 ; midstream urine, normal ; blood urea, 40 ; serum protein electrophoresis: increase in gamma-globulin and slightly reduced albumin; liver function tests, S.G.P.T. 10 ; alkaline phosphatase, $5 \mathrm{~K}$-A units; thymol turbidity, 3.1 units; zinc sulphate turbidity, 22.2 Kunkel units. The findings in respiratory function tests were consistent with hyperventilation associated with a combination of pulmonary fibrosis and ventilatory obstruction. Radiographs of the chest showed bilateral diaphragmatic elevation with partial atelectasis at both bases.

\section{COMMENT}

The effect of pregnancy on systemic lupus erythematosus has been excellently reviewed by Garsenstein et al. (1962), Murray (1958), and Mund et al. (1963). Friedman and Rutherford (1956) reported on the relationship of pregnancy to systemic lupus erythematosus. It appeared that fertility was not affected; the abortion rate was increased to $15.8 \%$-approximately twice 